\title{
Number of Zeros of a Polynomial (Lacunary-type) in a Disk
}

Idrees Qasim, Tawheeda Rasool and Abdul Liman

ABstract: The problem of finding out the region which contains all or a prescribed number of zeros of a polynomial $P(z):=\sum_{j=0}^{n} a_{j} z^{j}$ has a long history and dates back to the earliest days when the geometrical representation of complex numbers was introduced. In this paper, we present certain results concerning the location of the zeros of Lacunarytype polynomials $P(z):=a_{0}+\sum_{j=\mu}^{n} a_{j} z^{j}$ in a disc centered at the origin. 